\title{
A Basic Study of the Adaptive Particle Swarm Optimization
}

\author{
Genki Ueno, Nobuhiro Iwasaki and Keiichiro Yasuda \\ Dept. of Electrical Engineering, Tokyo Metropolitan University \\ 1-1, Minamiosawa, Hachioji-shi, Tokyo 192-0397, Japan \\ E-mail: yasuda@eei.metro-u.ac.jp
}

\begin{abstract}
It is well known that Particle Swarm Optimization (PSO), which was originally proposed by J. Kennedy et al., is a powerful algorithm for solving unconstrained and constrained global optimization problems. Appropriate adjustment of its parameters, however, requires a lot of time and labor when PSO is applied to real optimization problems. Therefore we have so far indicated that meta-heuristics should have robustness and adaptability from the engineering viewpoint and have proposed an adaptive algorithm.

In this paper, we extend the parameters of PSO in order to realize advanced adaptability, and we treat the selection strategy of global information gbest as a new parameter. Furthermore we propose an adaptive Particle Swarm Optimization method using a new parameter to accomplish effective search in global optimization. Some numerical simulations are carried out in order to examine the adaptability of the proposed approach.
\end{abstract}

\section{Introduction}

Meta-heuristics is a paradigm of optimization techniques that can obtain a solution that has enough optimality within a practical calculation time.

Meta-heuristics usually has some parameters to be adjusted. It can solve various optimization problems efficiently by adjusting their parameters properly, which indicates that we can't solve a problem efficiently without adjusting their parameters properly.

Proper parameters are different in each problem, and finding proper parameters needs trial and error. Bearing application to engineering optimization problems in mind, it is undesirable to solve a problem many times to adjust parameters. It is important to obtain as good solution as possible in a practical calculation time .

Therefore we have so far indicated that metaheuristics should have the robustness and the adaptability from the engineering viewpoint and have proposed an adaptive algorithm of Particle Swarm Optimization.

Although we have examined adjustability, robustness, and adaptability of parameters of PSO, it becomes important for realization of advanced adaptability to examine possibility of adaptation of PSO in the broader framework.
In this paper, while we will add some new elements to $\mathrm{PSO}$, we treat the elements as new parameters which should be tuned. We regard the selection method of information used for the search as one of the new parameters. We propose the algorithm which changes selection method of the information used for the search to escape from locally optimal solutions.

In this paper, we extend the parameters of PSO in order to realize advanced adaptability, and we treat the selection strategy of global information gbest as a new parameter. Furthermore we propose an adaptive Particle Swarm Optimization method using a new parameter to accomplish effective search in global optimization. Some numerical simulations are carried out in order to examine the adaptability of the proposed approach.

\section{Outline of Particle Swarm Optimization (PSO)}

\subsection{Background of PSO}

In this section, the background, the fundamental concept and the details of PSO are referred. As expressed in the name, the algorithm of PSO has a swarm composed of multi-particles. Each particle has its own position and velocity (transfer vector). The search by multi-particles distinguishes PSO from many optimization methods proposed so far. Each particle shares global information of gbest, and interaction among particles makes their search efficient. Although only simple operations compose the PSO algorithm, the PSO method can solve nonlinear and multipeaked optimization problems efficiently

\subsection{Algorithm of PSO}

Though two different types of algorithms exist - binary number and real number forms, PSO in a real number form is only analyzed in this paper. Although PSO was originally invented in the process of the research on simulating the movement of the swarm in the 2-dimensional space, PSO as an optimization method can work in the optional $n$-dimensional space.

Each particle has its own position $x$ and transfer vector $v$. And each particle also has its own pbest, or the best position visited so far, and all the particles share gbest, or the best position visited by all the particles so 


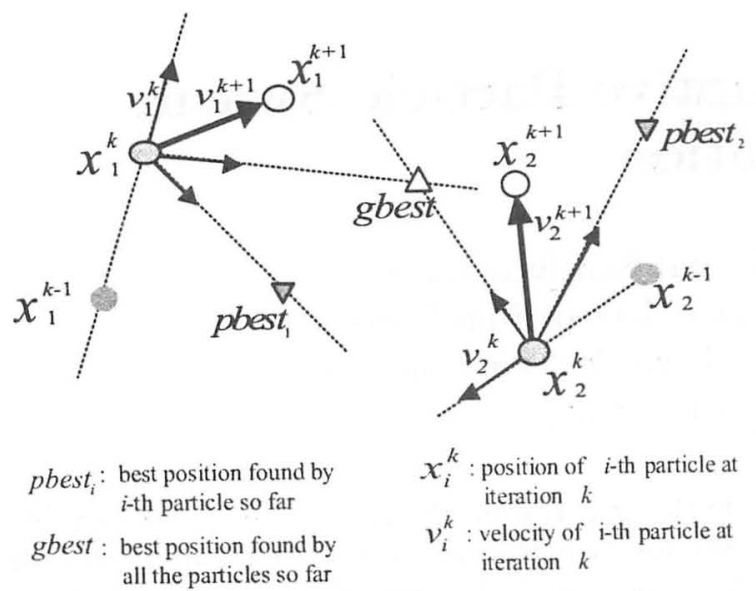

Fig. 1: Movement of particles

far. A particle forms a new transfer vector by linearly combining three vectors.

In the $(k+1)$-th transfer, the $j$-th coordinate component of transfer vector of the $i$-th particle is manipulated according to the following equation:

$$
\begin{aligned}
v_{i j}^{k+1} & =w \cdot v_{i j}^{k} \\
& +c_{1} \cdot \operatorname{rand}_{1}() \cdot\left(\text { pbest }_{i j}-x_{i j}^{k}\right) \\
& +c_{2} \cdot \operatorname{rand}_{2}() \cdot\left(\text { gbest }_{j}-x_{i j}^{k}\right)
\end{aligned}
$$

where $i=1, \cdots, m$ and $m$ is the size of the swarm; $j=$ $1, \cdots, n$ and $n$ is the size of space of a given problem; $w, c_{1}$ and $c_{2}$ are positive constants; $\operatorname{rand}()$ is random number which is uniformly distributed in $[0,1]$; and $k$ determines the iteration number.

Customarily, the transfer vector $v_{i j}$ is also called velocity in PSO, therefore this paper follows this custom. Now each particle moves according to the following equation:

$$
x_{i j}^{k+1}=x_{i j}^{k}+v_{i j}^{k+1}
$$

\section{Robustness, Adaptability and Adjustability}

We think that meta-heuristics should have the following three abilities, i.e., robustness, adaptability and adjustability.

The robustness of an optimization method describes the degree to which the method can guarantee the performance of search for pre-adjusted parameters against the predetermined structural variation of problems to be solved. While Figure 3 shows the image of an algorithm which has the robustness, Figure 2 shows the image of conventional algorithms. Algorithms with the robustness can solve more problems than conventional algorithms. We can expect that algorithms which have

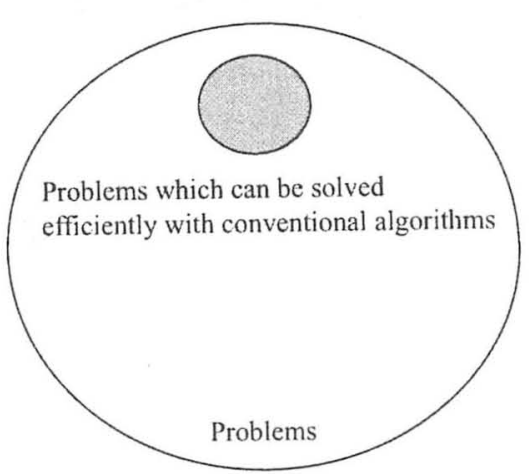

Fig. 2: Conventional algorithms

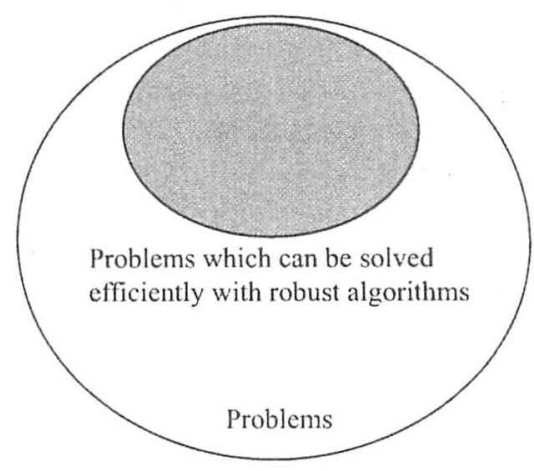

Fig. 3: Algorithms with robustness

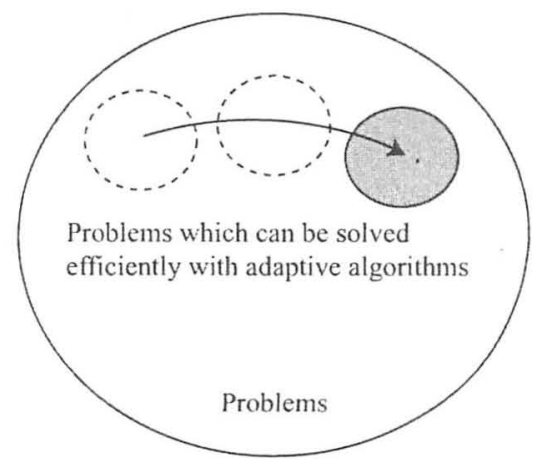

Fig. 4: Algorithms with adaptability 
the robustness will obtain a good solution for many problems without adjusting parameters strictly.

The adaptability of an optimization method describes the methods' ability to adaptively adjust its parameters against predetermined structural variation of the problem to be solved. Figure 4 shows the image of algorithms which have the adaptability. We can expect that algorithms which have the adaptability will obtain a good solution for many problems without adjusting parameters strictly because the algorithms can adjust their parameters by themselves.

The adjustability is an ability to guarantee the qualitative and quantitative relationship between parameters in an algorithm and the behavior of the algorithm.

And meta-heuristics with multiple search points such as PSO seems to have desirable conditions for adaptation because it can obtain much information from its searching process.

We have analyzed adjustability of parameters of PSO $w, c_{1}$ and $c_{2}$ and have proposed adaptive algorithm based on the analysis. Although we have examined adjustability, robustness and adaptability of parameters of PSO, it becomes important for the realization of advanced adaptability to examine possibility of adaptation of PSO in the broader framework.

\section{Extension of Parameters of PSO}

We have analyzed the adjustability of the parameters of PSO $\left(w, c_{1}\right.$ and $\left.c_{2}\right)$, and have proposed an adaptive PSO algorithm based on the analysis[5],[6],[7],[8]. We think, however, that there are other elements which can be tuned in PSO.

For example, information adopted as global information (gbest) or information adopted as local information (pbest), setting up different value for $w, c_{1}$ and $c_{2}$ of each particle, or construction of a group from some particles instead of construction of a group from all particles. Treating these elements as new parameters, we can extend the parameters of PSO.

Figure 5 shows the image of extension of parameters. Changing these new parameters, the behavior of search process of PSO will change. We verified that the parameter setting of conventional methods is not always best setting through some numerical simulations using a typical global optimization test problem.

Therefore if we establish a strategy for setting new parameters which have adaptability, the parameter setting will provide more effective search than conventional setting.

\section{Main Causes of Failure of Search}

The main causes of failure of search are as follows;

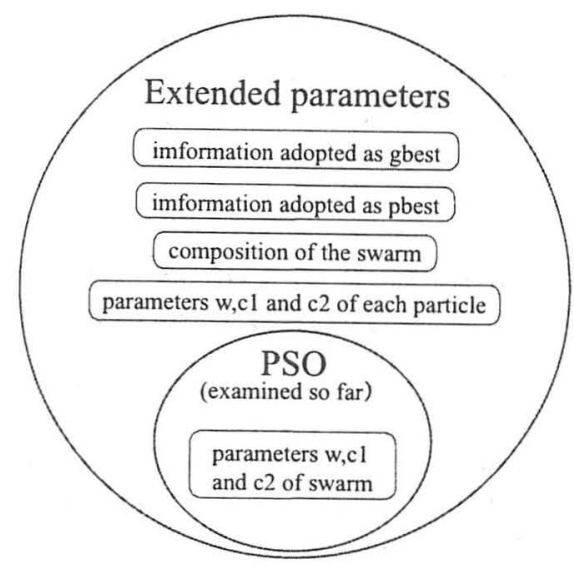

Fig. 5: Extension of parameters

(a) particles diverge to out of a feasible region or don't converge.

(b) particles converge to a point and stop moving.

(c) particles can not move out from a locally optimal solution.

Feature of convergence of the swarm is related to parameters $w, c_{1}$ and $c_{2}$. If feature of convergence of the swarm is not proper, phenomena such as above (a) or (b) happen and the swarm can't search efficiently. Figures 6, 7 and 8 show the example of above cases. The fitness of solution and the average of velocity of all particles are plotted.

In case (a), the parameters are too convergent and the movement of the particles nearly stops like Figure 6.

On the other hand, the parameters have divergent character, the average velocity gradually increases and the search ends in the failure in case (b). Or the swarm is not sufficiently concentrated, and the swarm can't search with accuracy.

In case (c), although the movement of the particles doesn't stop or diverges, fitness of solution is not improved in the long term like Figure 8. Even if feature of the convergence of the swarm is proper, these happen. We think that the cause of (c) is following phenomena.

Particles search neighborhood of own pbest and gbest. If gbest is near to a locally optimal solution and there are no other good solutions near current gbest, it is difficult to improve the fitness of solution. And if the fitness of gbest is not improved, the position of gbest doesn't change and search space of swarm is difficult to change. Therefore particles don't escape locally optimal solution.

It is necessary to adjust the feature of the convergence of the swarm properly. However even if we adjust the feature of the convergence properly, PSO can't always search efficiently because the phenomenon like above 
(c) sometimes happens. Therefore it is important for realization of more effective search to make the method in which particles escape locally optimal solution or don't converge to near locally optimal solution.

\section{Modification of Global Information of PSO (gbest)}

In PSO, particles use its own pbest and swarm's gbest. We usually use the best position visited by the particle so far as pbest and the best position visited by all the particles so far as gbest. We think that PSO searches efficiently because it uses information of good solution visited by the particles so far.

pbest and gbest are, however, parts of good solutions which the particles visited so far. The swarm has many other information of good solution. We think that it is effective for search to use those information. It can be thought that each good solution is near to locally optimal solution.

We think that information of good solution far from current gbest is useful in order to escape locally optimal solution and search near other good solution. Therefore, we think the information which is adopted as gbest is a new parameter.

And we propose a method which uses not only information of best position visited by all the particles so far as gbest, but also information of other good solutions. In this method, when particles can not escape locally optimal solution, the swarm uses information of the other good solution as gbest to escape locally optimal solution.

\section{Proposed Adaptive Particle Swarm Optimization}

In this section, details of the method which we propose in this paper are referred.

In this method, following function is added to PSO. The swarm preserves the information of past pbest which is far from current gbest and has good fitness. when the improve rate during decided period is small, the information adopted as gbest is replaced with best one of the preserved information of pbest.

Figure 9 shows the image of proposed method. In Figure 9, circles show the search space of each particle. The search space of particles changes when the position of gbest is changed.

\section{【Algrithm of the proposed method】}

step 1 : The initial position $x_{i j}$ and velocity $v_{i j}$ of particles is determined with randamness.

step 2 : The fitness of solution where particles are in is estimated.

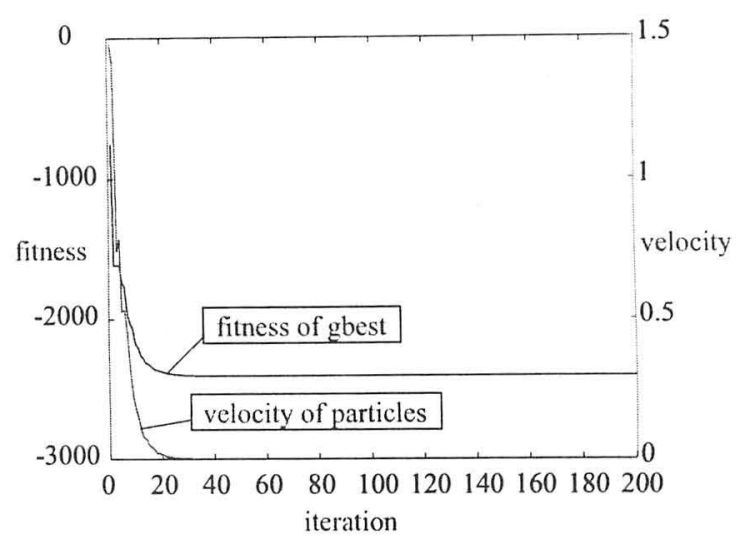

Fig. 6: An example of cașe(a)

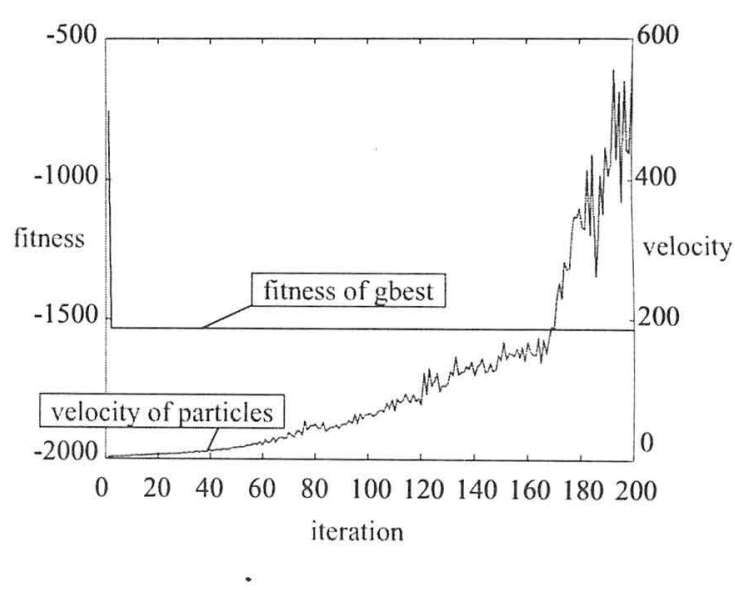

Fig. 7: An example of case(b)

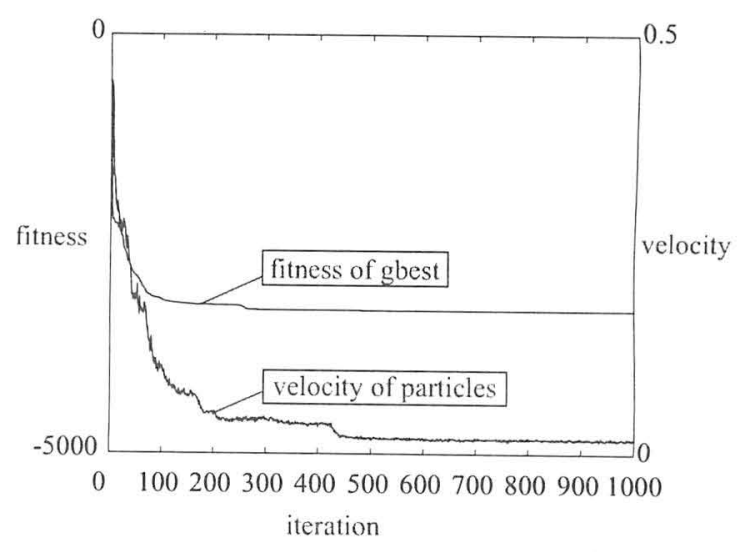

Fig. 8: An example of case(c) 
step 3 : The fitness of the current solution of each particle is compared with the fitness of own pbest. If the fitness of the current solution of each particle is better, pbest is replaced with the current solution of particles.

step 4 : The fitness of each particle's pbest is compared with swarm's gbest. If the fitness of pbest is better, gbest is replaced with the pbest.

step 5 : The information of pbest which is far from current gbest and has good fitness is preserved.

step $6: v_{i j}$ and $x_{i j}$ of the particles are renewed using Equations (1) and (2).

step 7 : When the improvement rate of gbest during decided period is small, the position of gbest is moved to the position which has best fitness in the preserved information.

step 8 : Steps 2-7 are iterated until predetermined iteration number

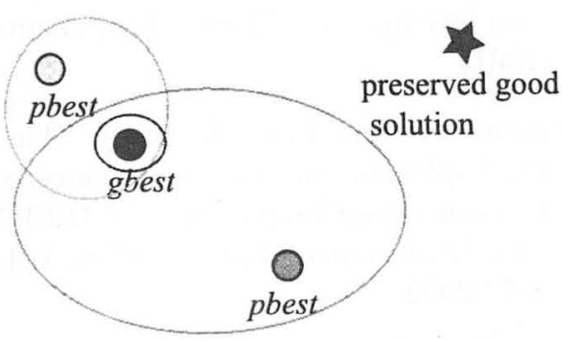

Modification of imformation adopted as gbest

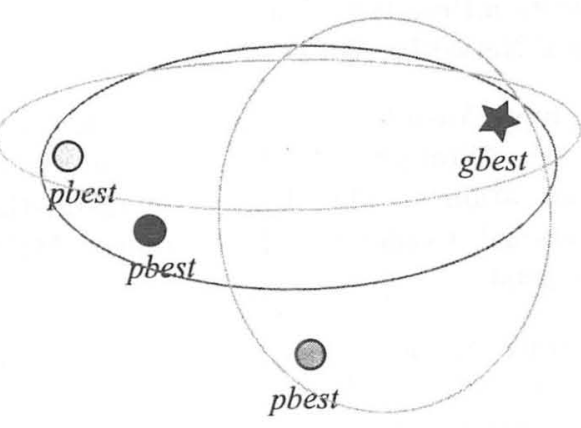

Fig. 9: Image of the proposed method

\section{Numerical Simulations and Considerations}

We verify the search ability of the proposed method by the numerical simulation using typical benchmark problems.

\subsection{Numerical Simulations}

We compare the proposed method with the conventional PSO by the numerical simulation using two typical benchmark problems. The benchmark problems used in this paper are $2^{n}$ minima and rastrigin.

Figure 10 and Figure 11 show the shapes of the benchmark problems. These benchmark problems have many locally minimal solutions. Table 1 shows the conditions of each problem and results of application of the proposed method and the conventional PSO. The conditions of the above simulation are same at each method.

$$
\begin{aligned}
& \text { min } \quad f=\sum_{i=1}^{n} \text { minima] } \\
& \text { subj.to } \left.-5 \leq x_{i}^{4}-16 x_{i}^{2}+5 x_{i}\right) \\
& \text { [rastrigin] } \\
& \text { min } f=\sum_{i=1}^{n}\left(x_{i}^{2}-10 \cos 2 \pi x_{i}+10\right) \\
& \text { subj.to }-5 \leq x_{i} \leq 5
\end{aligned}
$$

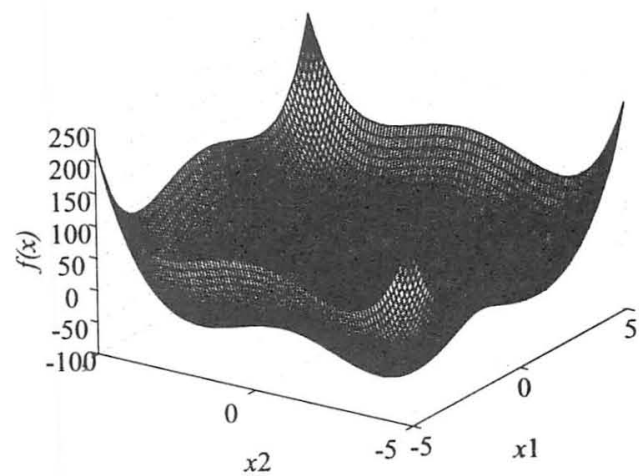

Fig. 10: Shape of $2^{n}$ minima function

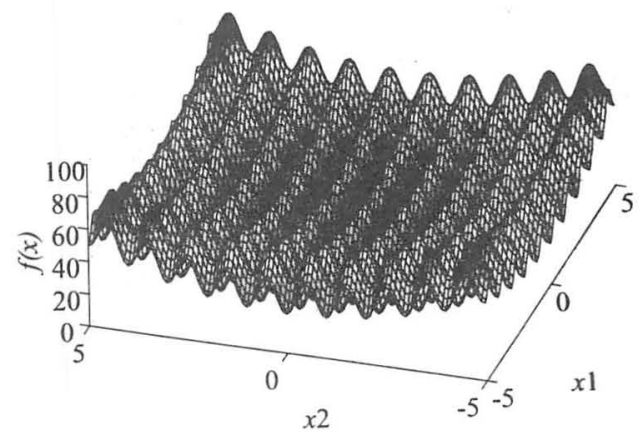

Fig. 11: Shape of rastrigin function 


\subsection{Considerations of Numerical Simulations}

Table 1 shows the results of the numerical simulations. The results of the proposed method are better than those of the conventional PSO for these numerical simulations. We verified that the proposed method is effective in some problems by these simulations.

And Figure 12 shows a good case of the proposed method and Figure 13 shows a good case of the conventional PSO. In these cases, the fitness of solutiond can not be improved sufficiently after iteration 40 . In the proposed method, information which is adopted as gbest was changed at iteration 65. And the fitness of solutions became worse temporarily and was improved after a few iterations. The fitness of solutions found by the proposed method is better than that found by the conventional PSO.

Table 1: Result of numerical simulations

\begin{tabular}{|c|c|c|c|c|}
\hline function & dim. & iteration & PSO & $\begin{array}{c}\text { proposed } \\
\text { method }\end{array}$ \\
\hline \hline $2^{n}$ minima & 10 & 100 & -721 & -708 \\
\hline $2^{n}$ minima & 10 & 500 & -769 & -759 \\
\hline rastrigin & 10 & 100 & 14.1 & 14.9 \\
\hline rastrigin & 10 & 500 & 8.0 & 9.1 \\
\hline
\end{tabular}

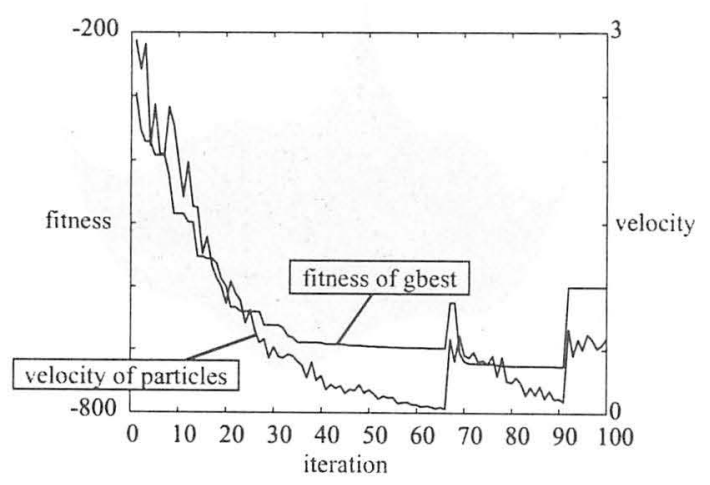

Fig. 12: Proposed method

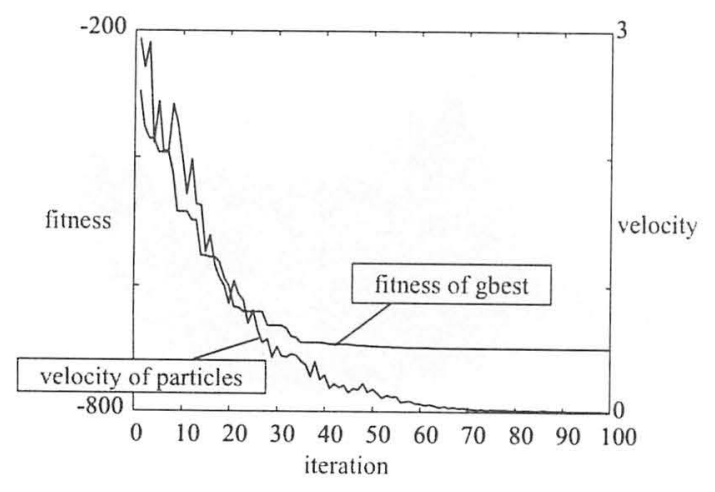

Fig. 13: Conventional PSO

\section{Conclusion}

In this paper, while we examined the robustness, adaptability and adjustability, we added new elements to PSO which can be tuned as new parameters.

We regarded the selection method of information gbest as a new parameter. We proposed a method which changes selection method of the information gbest in order to escape a locally optimal solution. In this method when particles can not escape from a locally optimal solution, the swarm uses information of other good solution as gbest in order to escape from a locally optimal solution.

And we verified the search ability of the proposed method by the simulations using typical benchmark problems.

\section{References}

[1] James Kennedy and Russell C. Eberhart : "Swarm Intelligence", Morgan Kaufmann Publishers (2001)

[2] Maurice Clerc and James Kennedy, "The Particle Swarm-Explosion, Stability, and Convergence in a Multidimensional Complex Space", IEEE Transactions on Evolutionary Computation, Vol.6, No.1, pp.58-73 2002.

[3] Y. Shi, R.C. Eberhart, "A modified particle swarm optimizer", Proceedings of the IEEE Congress on Evolutionary Computation (CEC 1998), Piscataway, NJ. pp. 69-73, (1998)

[4] J.Kennedy and R.Everhart : Particle Swarm Optimization,Proc.IEEE International Conference on Neural Networks, Vol.IV,(1995)

[5] Keiichiro Yasuda, Azuma Ide and Nobuhiro Iwasaki, "Stability Analysis of Particle Swarm Optimization", The Fifth Metaheuristics International Conference Proceedings, MIC03-34, (Aug.2003)

[6] Keiichiro Yasuda, Azuma Ide and Nobuhiro Iwasaki, "Adaptive Particle Swarm Optimization", IEEE International Conference on Systems, Man \& Cybernetics Proceedings, TA8-1, pp.1554-1559, (Oct.2003)

[7] K. Yasuda :"Evolutionary Computation and Metaheuristics", T.IEE Japan, Vol.122-C, No.3, pp.320$323(2002)$

[8] K. Yasuda and N. Iwasaki: "Adaptive Particle Swarm Optimization using Velocity Information of Swarm", 2004 IEEE International Conference on Systems, Man and Cybernetics (2004) 